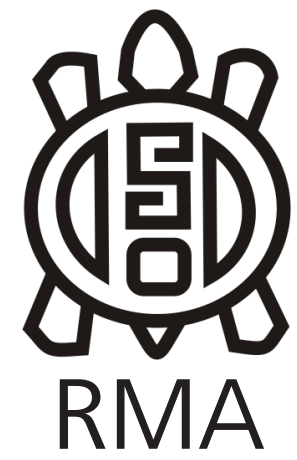

Dossier

\section{En busca de un acuerdo sobre aspectos básicos para el estudio tafonómico de conjuntos líticos}

\author{
In pursuit of an agreement on basic aspects for the taphonomic study \\ of lithic assemblages
}

Catalina Balirán*

*Facultad de Filosofía y Letras - Universidad de Buenos Aires. CABA, Argentina. E-mail: cataclorofila@gmail.com

\title{
Resumen
}

En los últimos años se ha incrementado la aplicación de estudios tafonómicos al registro arqueológico lítico y se ha demostrado no solo su utilidad para conocer los procesos de formación posdepositacionales, sino también, su importancia para una mejor comprensión e interpretación de las conductas humanas en el pasado. Dado esto, el presente trabajo se propone hacer una síntesis que abarca cuestiones relativas a cuatro etapas de la planificación y realización de un estudio tafonómico centrado en el registro lítico: 1) planteo del tema a ser estudiado, que incluye la identificación del problema específico de investigación, el tipo de registro arqueológico, los agentes tafonómicos y las escalas de trabajo, entre otros; 2) análisis de los conjuntos y planteo de nuevas hipótesis; 3) testeo de hipótesis; y 4) interpretación. Asimismo, se enfatiza el rol de la experimentación como vía actualística clave para el desarrollo de los estudios tafonómicos.

Palabras clave: Arqueología; Tafonomía; Lítico; procesos de formación; experimentación.

\begin{abstract}
In recent years the application of taphonomy to the study of the lithic archaeological record has increased and it proved to be a significant contribution to the understanding of past human behavior. Within this context, the present paper synthesize a four-stage proposal for taphonomic studies focused on the lithic record: 1) definition of the research problem which includes the identification of: the specific research question, the archaeological context, the taphonomic agents and the scales of analysis, among others; 2) analysis of lithic samples and the definition of new hypotheses; 3) testing of hypotheses; and 4) interpretation. Also, the role of experimentation as a key actualistic element for the development of taphonomic approach is emphasized.
\end{abstract}

Keywords: Archaeology; Taphonomy; Lithic, Formation Processes; Experimentation.

\section{Introducción}

Si bien el estudio de los procesos de formación y su efecto sobre el registro arqueológico lítico tiene larga data (Barnes, 1939; Mason, 1965; Rick, 1976; Wilk y Schiffer, 1979 entre otros), recién a mediados de la década de 1980 se planteó explícitamente la necesidad de aplicar una perspectiva tafonómica al estudio de los materiales líticos (Hiscock, 1985). Hiscock argumentó con evidencia empírica que los mismos procesos que afectan al material óseo también alteraban el estado de los artefactos de piedra y que los efectos de dichos procesos podían ser medidos e interpretados. En Argentina fue Borrazzo (2006) quien introdujo la tafonomía a los estudios líticos y aplicó esta perspectiva al registro procedente de distintos ambientes donde predomina la acción de diversos agentes tafonómicos (Borrazzo, 2006, 2010; Weitzel, Borrazo, Ceraso, y Balirán, 2014 entre muchos otros). Ella definió a la tafonomía lítica como: "el estudio arqueológico y actualístico que describe, define y sistematiza los efectos producidos por los agentes y procesos naturales y culturales que actuaron sobre los conjuntos artefactuales líticos con posterioridad a su depositación y hasta el momento de su recuperación en el contexto arqueológico" (Borrazzo 2006:249). En los últimos años, esta perspectiva ha comenzado a ser utilizada por otros investigadores del cono Sur (Balirán, 2014; Carranza Elola, 2015; Carranza, 2015; Cañete Mastrángelo y Muñoz, 2018; Méndez Muñoz, 2015; Ozán, 2017; Somonte y Baied, 2013; Ugalde et al., 2015). Estos trabajos han demostrado no solo la utilidad del enfoque tafonómico para entender los procesos de formación del registro, sino también la importancia de aplicarlos para generar interpretaciones válidas acerca del pasado.

Este trabajo busca compendiar los pasos generales que 
componen los programas de investigación tafonómicos del registro lítico. En este sentido, el objetivo es extraer y sintetizar aquí aquellos lineamientos o aspectos comunes entre los trabajos tafonómicos de materiales líticos disponibles, independientemente de los contextos arqueológicos u objetivos de investigación diferentes de cada uno de ellos.

\section{Algunos aspectos básicos}

Un abordaje tafonómico del registro lítico implica el estudio de las variaciones espaciales y morfológicas que sufren los artefactos desde su depositación hasta que son recuperados para su estudio (Schiffer, 1987). La intensidad con la que estas variaciones suceden puede afectar el registro de forma tal que, si pudiéramos tomar una fotografía del aspecto y organización de los artefactos en el momento de su depositación inicial y otra durante su recuperación arqueológica, encontraríamos que pueden ser muy distintas entre sí o simplemente dejaron de existir. El registro se presenta de forma continua con una densidad más o menos variable y nuestro trabajo será determinar a qué pueden deberse esas variaciones (Dunnell, 1992). En este sentido es útil considerar al registro arqueológico como un palimpsesto que puede presentar diversas características relacionadas con cuestiones temporales, espaciales o de significado (Bailey, 2007) debido a que esta es la forma más usual (sino la única) en la que se presenta el registro arqueológico (Borrero, 2014a). Si bien usualmente la presencia de palimpsestos es vista como una desventaja del registro arqueológico que es necesario superar, alternativamente puede pensarse en los palimpsestos como una ventaja que permite focalizarse en escalas del comportamiento diferentes a las que se observan a nivel etnográfico (Bailey, 2007; Binford, 1981). Comprender las dinámicas formacionales que dan lugar a la creación de palimpsestos resulta a su vez crucial para encarar cualquier tipo de interpretación del registro arqueológico (Davies, Holdaway, y Fanning, 2016; Fanning, Holdaway, Rhodes, y Bryant, 2009). Adicionalmente los estudios tafonómicos requieren del uso de distintas escalas de análisis, tanto temporales como espaciales (Dincauze, 1987) ya que la naturaleza de los distintos agentes tafonómicos así puede requerirlo. En este sentido la tafonomía regional, que permite la identificación de modos tafonómicos, resulta clave para la detección y medición de agentes tafonómicos y la comparabilidad entre distintos espacios con características ambientales y preservacionales similares (Borrazzo y Borrero, 2016; Borrazzo y Borrero, 2015; Borrero, 2001, 2014b).

Un último concepto central es el de uniformitarismo metodológico. Este acercamiento propone que ciertas propiedades, características o procesos del universo se mantienen constantes o uniformes en el tiempo y el espacio, y, que el tratamiento de los mismos debe ser intelectualmente independiente de aquello que deseamos investigar (Ascher, 1961; Domínguez-Rodrigo, 2008; Gifford-Gonzalez, 1981; Lin, Rezek, y Dibble, 2018). Por ejemplo, para el caso de una roca que contenga un lascado aislado, puede suponerse que la mecánica de fractura de las rocas en el pasado es la misma que en el presente, y que, a su vez, muchos de los agentes que pudieron producirlas en el pasado, pueden ser observados en el presente o sus acciones ser reproducidas en la actualidad de forma experimental. Sin embargo, no podríamos asumir a priori que determinada fractura haya sido causada de forma intencional (o no) por determinado agente o proceso. Dicho de otro modo, las leyes que gobiernan la mecánica de fracturas existen independientemente de quién o qué aplique la fuerza necesaria para producirlas. Esto último enfatiza el rol clave del estado del conocimiento y la aplicación de ese conocimiento que realiza cada analista lítico.

\section{Esquema de trabajo tafonómico}

A continuación, se propone una estructura a partir de cómo hasta ahora se ha planificado, pensado y trabajado con una perspectiva tafonómica (Figura 1). Este esquema no es una forma última ni mucho menos acabada de cómo se debe trabajar en tafonomía, sino una propuesta que ha sido de utilidad en la planificación y desarrollo de otros trabajos y que sin duda debe ser adecuada a diversos casos de estudio (Carranza Elola, 2015; Carranza, 2015; Cañete Mastrángelo, D. S., \& Muñoz, A.S. 2018; Méndez Muñoz, 2015; Somonte y Baied, 2013; Ugalde et al., 2015 entre otros). Los puntos de este esquema están planteados a partir de en una serie de preguntas que suelen ser relevantes en este tipo de investigaciones. En el presente trabajo, el esquema está dividido en cuatro etapas o momentos de la investigación: 1) planteo del tema a ser estudiado; 2) análisis de los conjuntos y planteo de nuevas hipótesis; 3) testeo de hipótesis; y 4) interpretación.

\section{Planteo del tema de estudio}

Identificación del problema.

¿Cuál es el problema? Esta es una pregunta que funciona a varios niveles, y es la primera que suele aparecer (Punto 1 en figura 1). Por un lado, se refiere al problema arqueológico general sobre el cual se trabaja (por ejemplo, movilidad de grupos, aprovisionamiento de materias primas, etc.). Por otro lado, incluye también los problemas formacionales que se identifican en el área de trabajo y que pueden afectar en alguna escala el registro estudiado (Schiffer, 1987). Dicho de otra forma, se trata de identificar procesos y agentes tafonómicos. En este punto es muy importante la interrelación que existe entre ambos aspectos de la pregunta, ya que, mientras el registro arqueológico es afectado por diversos agentes tafonómicos, estos funcionan a diferentes escalas, tanto espaciales como temporales, y con diferentes intensidades. Cuando las escalas en las que se trabaja son 
definidas, es útil no perder de vista las escalas en las que actúan los agentes tafonómicos, y lo mismo a la inversa. De esta observación simultánea tanto de los problemas arqueológicos como de los aspectos formacionales, pueden surgir las primeras preguntas tafonómicas. Dicho de otro modo, se pueden generar hipótesis de tipo general sobre qué efectos son esperables en el registro bajo estudio, a partir de la acción de los agentes tafonómicos observados.

Identificación del tipo de registro.

En concordancia con lo recién planteado, surge la siguiente pregunta: ¿cómo se presenta el registro con el que se trabaja? Esta pregunta apunta principalmente a pensar los tipos de procesos que afectan al registro arqueológico, según se encuentre éste en superficie o en estratigrafía, en este sentido el estudio de la matriz sedimentaria desde una perspectiva geoarqueológica puede ser de gran utilidad (Fanning, Holdaway, Rhodes, y Bryant, 2009). Este paso sirve para dimensionar los procesos y agentes tafonómicos que se estudian, sin olvidar que aquello que está enterrado alguna vez estuvo en la superficie y, en muchas oportunidades, lo contrario también ha sido el caso (Binford, 1992) (Punto 2 en figura 1).

Identificación de agentes tafonómicos.

Teniendo en cuenta lo anterior ¿cuáles son o fueron los procesos o agentes tafonómicos que pudieron estar, o están presentes, y afectaron o afectan el registro arqueológico bajo estudio? Esto conlleva la reducción del universo de agentes tafonómicos identificados en el primer paso de este esquema a aquellos relevantes para el estudio que se realiza (Punto 3 en figura 1). Por ejemplo, la existencia de procesos glaciares en el área trabajada cuya cronología es anterior a la de la depositación del registro estudiado, carecería de interés para el análisis tafonómico del conjunto bajo estudio.

Identificación de escalas y consecuencias observacionales. Una vez identificados los agentes tafonómicos que pudieron afectar o afectan el registro bajo estudio, lo que sigue sería pensar ¿en qué escala son identificables? Y ¿Cómo afectan o pudieron afectar al registro arqueológico los agentes tafonómicos identificados? (Punto 4 en figura 1). Ambas preguntas componen un mismo momento de la investigación. La primera de las preguntas apunta a destacar que, si bien los agentes y procesos tafonómicos siempre afectan a los artefactos, a veces su acción se hace identificable o visible a escala de los conjuntos. Por ejemplo, cuando se trata de la acción de un curso de agua sobre un conjunto lítico, la fuerza del agua moviliza a cada artefacto de forma individual, pero para identificar si existe una selección granulométrica -lo que permitirá hipotetizar sobre la acción de dicho proceso- es necesario hacer una evaluación a nivel del conjunto. La segunda pregunta apunta a identificar las consecuencias observacionales de la acción de dichos agentes. En este punto suelen aparecer, ya no en términos generales sino de forma específica, las primeras hipótesis tafonómicas. Esto permite delinear qué variables (e.g .meteorización química, corrasión, fragmentación, rock coatings) pueden resultar relevantes en función de estas hipótesis. Asimismo, en esta etapa suele ser posible identificar qué variables podrán ser registradas a escala de los artefactos y cuáles a escala de los conjuntos (Punto 5 en figura 1).

\section{Análisis de los conjuntos artefactuales}

¿Qué hacer con toda esta información? Este es el momento de la investigación en el que suelen realizarse los análisis tafonómico y tecnológico de la muestra (Punto 6 en figura 1). Para ello, ya identificados los agentes tafonómicos y las consecuencias observacionales de su accionar sobre el registro estudiado (efectos tafonómicos, Lyman 1994), es crucial definir aquellas variables a observar y la forma en la que las mismas se harán operativas, es decir, cómo serán registradas. Variables sobre las cuales (meteorización química, corrasión; fragmentación; etc.) se focalizará el análisis tafonómico de los artefactos.

El análisis puede brindar un nuevo set de hipótesis tafonómicas que surjan de la exploración de los datos, ya que, a partir del cruce de las distintas variables (tafonómicas y tecnológicas) se pueden observar patrones a nivel del conjunto que antes no eran evidentes (Punto 7 en figura 1). Asimismo, a partir del análisis tecnológico surgirán las hipótesis sobre uso y estrategias tecnológicas. En los casos en los que se ha utilizado un enfoque tafonómico, las interpretaciones del registro arqueológico se han visto beneficiadas ya que, además de permitir una mejor comprensión de los procesos posdepositacionales, este enfoque ayudó a evitar la sobreestimación de la agencia humana en la variabilidad material observada.

\section{Testeo de hipótesis}

Por ultimo, pero no menos importante: ¿es posible testear las hipótesis tafonómicas? La respuesta recurrente en los trabajos es que sí es posible y, en general, muchos autores han seguido la vía de los estudios actualísticos para hacer esa evaluación (Puntos 8 y 9 en figura 1). Existen diversas formas de testeo de hipótesis, en general apuntadas a generar cuerpos de datos, los cuales son empleados como marco de referencia a contrastar con el registro arqueológico. Las observaciones naturalistas y la experimentación suelen ser dos formas muy utilizadas (Punto 10 en figura 1). La vía elegida puede depender del tipo de agente tafonómico que se busque evaluar. La acción de agentes de escala masiva, como por ejemplo la acción marina o glaciar, es difícil de reproducir experimentalmente, por lo que en estos casos las observaciones naturalistas permiten acceder, desde casos actuales y ambientes conocidos, a información que puede ser aplicable a casos de estudio arqueológicos. 


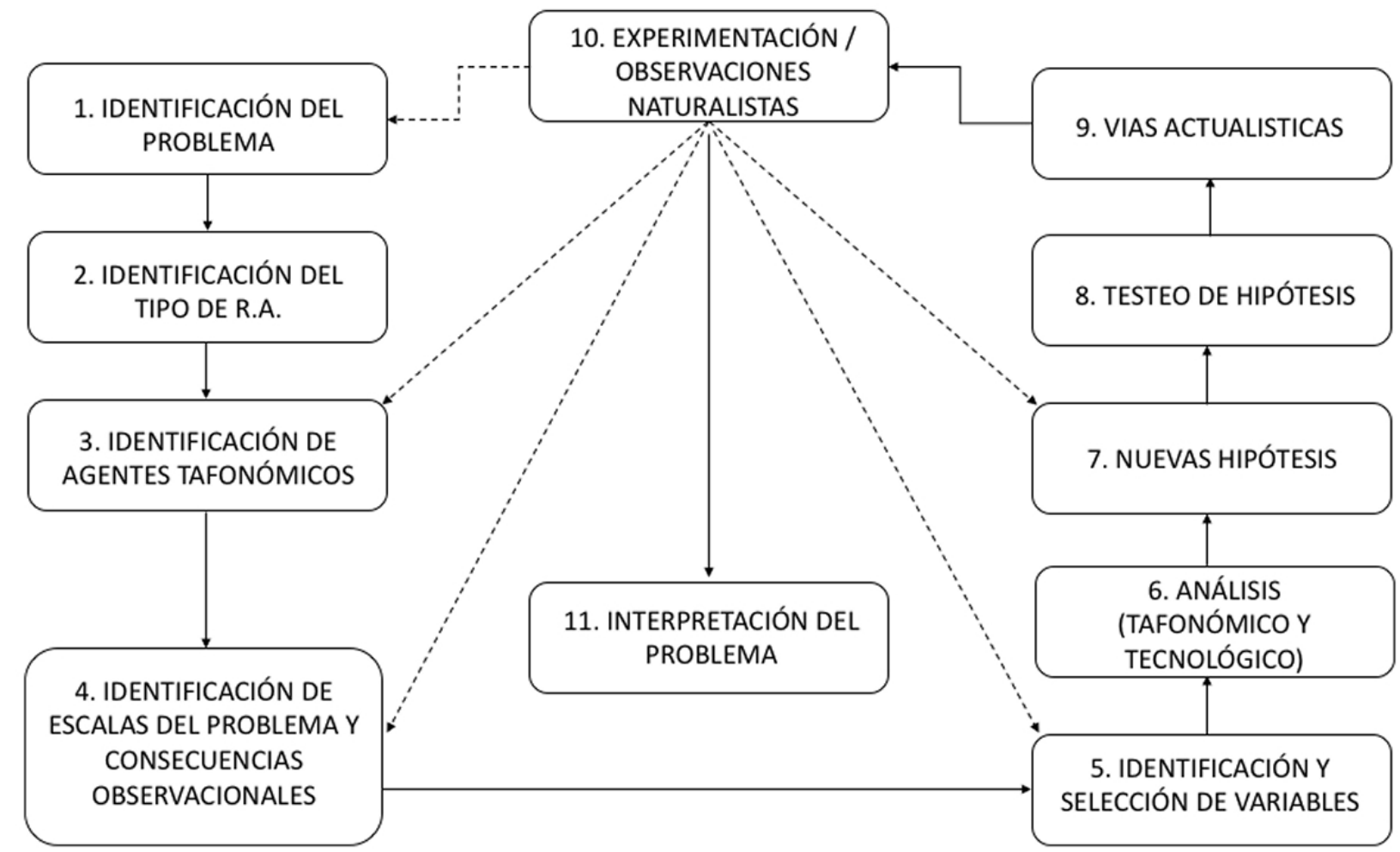

Figura 1. Esquema presentado en el texto. Las flechas continuas representan el orden inicial de trabajo. Las flechas puntuadas indican algunos de los pasos del esquema a los que se puede volver con información nueva obtenida del mismo proceso de trabajo.

Figure 1. Scheme presented in the article. The solid arrows represent the initial workflow. The dotted arrows indicate some of the steps to which it is possible to return with new information obtained from the initial workflow

Sin embargo, mientras que la reproducción de procesos visibles en grandes escalas temporales o espaciales puede revestir algunas dificultades, es factible replicar procesos derivados de aquellos de escala masiva o bien la acción de otros agentes que actúan a escalas menores. En este sentido la experimentación ha demostrado ser una vía útil de trabajo (Carranza, 2015; Eren et al., 2010; Flegenheimer y Weitzel, 2007; Lopinot y Ray, 2007; Méndez Muñoz, 2015). Las principales ventajas de la experimentación para la evaluación de hipótesis arqueológicas en general y tafonómicas en particular residen principalmente en que, un experimento bien diseñado brinda información que puede llenar ese vacío que existe entre el fenómeno observado y nuestras hipótesis (Nami, 2011, 2018). Adicionalmente su costo puede ser relativamente bajo, lo que aumenta la factibilidad de su realización.

Interpretación del problema: algunas cuestiones sobren experimentación

Sin embargo, no todo experimento produce información relevante (Borrero, 1991) y no toda inferencia es válida. La validez de las inferencias depende directamente del diseño experimental (Domínguez-Rodrigo, 2008). Los experimentos deben estar diseñados de forma tal que fortalezcan la validez de las inferencias ${ }^{1}$ que de ellos

1 Entendemos el concepto de validez de las inferencias derivan. Así las mismas podrán ser utilizadas de forma segura para la interpretación arqueológica (Punto 11 en figura 1).

La validez externa de un experimento se alcanza cuando sus resultados puedan ser generalizados y aplicados a un rango relativamente amplio de contextos. La validez interna, por su parte, depende de la precisión del experimento en el contexto experimental, donde debe ser posible determinar cuál de las variables independientes es la responsable de la variación en las variables dependientes bajo estudio. De este modo, cuanto mejor se replique a nivel experimental el contexto arqueológico que nos interesa comprender, más bajo será el nivel de error (Lin et al., 2018).

La primera cuestión a tener en cuenta al momento de diseñar un experimento es el control. Tanto las hipótesis de trabajo como las expectativas deben ser explicitadas de antemano y son las que guiarán el diseño experimental. De ello depende el establecimiento de cuáles son las variables a controlar. Respecto de esto, Borrero (1991) advierte que no siempre es posible tener un control absoluto sobre las

como "our ability to be confident about the causal nature of the stipulated relationship and the generalizability of this relationship to archaeological material" (Lin et al., 2018:676). 
variables del experimento. En el caso de los experimentos que no contemplan un registro constante (por ejemplo, pistas experimentales con seguimiento longitudinal periódico), se conocen las condiciones iniciales y las condiciones al momento del, o los, relevamientos y lo que sucede en el medio se transforma en una caja negra. Cuando esto es inevitable, debe informarse cuáles son los aspectos que quedarán fuera de control para evitar analogías que no son sustentadas adecuadamente. Una de las formas de contrarrestar el efecto de las cajas negras es plantear varias pistas o cuadrículas experimentales en diferentes localizaciones que presenten condiciones similares y luego establecer el rango de resultados posibles. En segundo lugar, el diseño debe explicitar las hipótesis a testear. En este sentido Domínguez-Rodrigo (2008) afirma que los "experimentos realizados para testear una hipótesis deben ser capaces de testear su opuesta y refutarla" (Domínguez-Rodrigo 2008:74). Por lo tanto, la hipótesis nula también debe hacerse explícita. Por último, para mantener la validez externa e interna del experimento deben considerarse algunas cuestiones que garanticen la comparabilidad de la muestra experimental con el registro arqueológico bajo estudio: 1) el contexto en que se realiza el experimento debe ser equivalente o poder replicar (en la medida de lo posible) el contexto arqueológico bajo estudio en términos ecológicos y geomorfológicos; 2) la muestra experimental debe ser adecuada para su comparabilidad en términos de materia prima, composición y tamaño de los artefactos experimentales; 3) identificar de forma clara las variables independientes y las variables dependientes (DomínguezRodrigo 2008).

En relación a estos puntos, Ascher (1961) señala que existen tres cuestiones que dirigen o limitan un experimento: 1) que nuestro objeto de estudio (e.g. determinada materia prima) haya estado disponible en los contextos pasados que estudiamos; 2) disponibilidad presente o pasada de los agentes considerados responsables del fenómeno observado; 3) trabajar dentro de las limitaciones de las primeras dos (cualidades de los materiales y los agentes y/o procesos tafonómicos) (Ascher, 1961). Estas cuestiones permiten comenzar con el delineado de las variables que son necesarias controlar, como así también permiten identificar y explicitar aquellas que no serán controlables.

Finalmente, respecto del alcance explicativo, los experimentos que no registran cambios son experimentos positivos, y solo tienen valor para la escala temporal en la que han sido planteados. De ninguna manera esa ausencia de cambios puede ser extrapolada al largo plazo o escala temporal arqueológica. Alternativamente, se llama experimento negativo a aquel que registra cambios, $y$, su alcance explicativo se limita a los parámetros registrados en dichos cambios. Así, si en el corto plazo el experimento registra cambios, podemos esperar esos cambios en el largo plazo (Borrero 1991). Sin embargo, los resultados obtenidos en los plazos experimentales no pueden ser extrapolados de manera lineal a los tiempos arqueológicos.

\section{A modo de ejemplo}

En el contexto del estudio de la circulación de poblaciones cazadoras recolectoras y uso del espacio en el sur de Patagonia continental se recuperaron artefactos líticos en contexto de superficie de la localidad arqueológica La Verdadera Argentina (LVA, Santa Cruz, Argentina). Los artefactos recuperados presentaban en su superficie distintos estadios de meteorización (i.e. alteración química), coincidiendo los más "frescos" con los lascados del filo de las piezas. Estas características pueden ser interpretadas como producto del proceso de reclamación (sensu Schiffer 1987). Sin embargo, las observaciones naturalistas en el área permitieron reconocer que estos artefactos, al estar expuestos en superficie, tenían una alta probabilidad de ser pisoteados por animales. A partir de estas observaciones se planteó como hipótesis alternativa que las características observadas en los artefactos eran producto del pisoteo. En función de ello, el análisis tafonómico de los conjuntos relevó variables que permitan conocer las condiciones a las que estuvieron expuestos estos conjuntos: estado (entera o fragmentada); presencia, extensión e intensidad de meteorización, y presencia, localización y extensión de barniz de suelo. Un dato que fue clave para los estudios tafonómicos fue el registro de la cara expuesta de cada artefacto al momento de su recuperación, ya que permitió evaluar condiciones de estabilidad (e.g. Borrazzo 2011). El análisis tafonómico indicó que se trataba de una muestra altamente fragmentada y que el menor grado de meteorización observado en ca. $50 \%$ de las piezas coincidía con el filo de las mismas. Dados estos resultados, se diseñó un experimento para evaluar la hipótesis alternativa (origen tafonómico de los filos más "frescos"). Los experimentos mostraron que las condiciones ambientales (presencia de fauna silvestre y ganado) disponibles en LVA producen el fenómeno observado como producto del pisoteo animal. Si bien estos resultados no son suficientes para refutar la hipótesis antrópica, señalan a los procesos posdepositacionales como una causa potencial del fenómeno observado (Balirán 2014).

\section{Palabras finales}

Este trabajo sintetizó un esquema de trabajo con perspectiva tafonómica posible a partir de la lectura, en clave metodológica, de trabajos que aplican este tipo de enfoques y han obtenido resultados positivos. Este esquema o estructura que presentamos aquí no representa la única forma de trabajo posible en tafonomía lítica y sin duda debe ser adecuado a los diferentes contextos de trabajo que se aborden. Por otro lado, se enfatizó la importancia del diseño y la planificación, sobre todo en casos donde la experimentación surja como una vía para 
evaluar de hipótesis, ya que esto permite optimizar las visitas al campo y los recursos económicos, muchas veces escasos para los investigadores. Consideramos importante señalar que, a pesar de su importancia, ni la tafonomía ni la experimentación son o deben ser un fin en sí mismos. Ambas aproximaciones responden a una pregunta arqueológica y nos permiten entender cómo los procesos tafonómicos pueden influir en nuestras interpretaciones del registro arqueológico. En este sentido creemos que es crucial entender que los estudios tafonómicos se potencian con los estudios tecnológicos: los primeros son una herramienta más de análisis que complementa a los segundos y en su integración permiten mejorar nuestras interpretaciones de la variabilidad observada en el registro lítico. Esto último hace a los estudios tafonómicos, no solo útiles, sino también necesarios.

Finalmente retomamos aquella propuesta de Binford (2001) de rechazar la utilidad del concepto de equifinalidad debido a que ella es un problema en nuestra capacidad de medición de los fenómenos. En este sentido consideramos que pensar a la equifinalidad como una ilusión asociada a las escalas con las que miramos y medimos los fenómenos del registro arqueológico puede servirnos como motivación para que los arqueólogos afinemos nuestros métodos de análisis, y, en definitiva, mejoremos nuestras interpretaciones del registro. Este trabajo sin duda es arduo pero no imposible, y creemos que la mejor vía para lograrlo será de forma conjunta entre investigadores. En este sentido esperamos que este trabajo sea un pequeño aporte a esa búsqueda colectiva.

Buenos Aires, 27 de Junio 2019.

\section{Agradecimientos}

A Karen Borrazzo y Luis Borrero, quienes, con su mirada crítica, me motivan a pensar y mejorar mi trabajo constantemente. A Eugenia Carranza y Federico Bobillo, con quienes en más de una oportunidad hemos podido discutir y reflexionar sobre estos temas que nos apasionan. A los organizadores del $1{ }^{\circ}$ Congreso Argentino de Estudios Líticos en Arqueología. Agradezco muy especialmente a los dos evaluadores anónimos por la lectura atenta y detallada de mi trabajo y cuyas sugerencias han sido de gran valor para mejorar el manuscrito original.

\section{Referencias citadas.}

Ascher, R. (1961). Analogy in archaeological interpretation. Southwestern Journal of Anthropology, 17(4), 317-325.

Bailey, G. (2007). Time perspectives, palimpsests and the archaeology of time. Journal of Anthropological Archaeology, 26(2), 198-223.

Balirán, C. (2014). Trampling, taphonomy, and experiments with lithic artifacts in the southeastern Baguales Range (Santa Cruz, Argentina). Intersecciones en Antropología,
15 (Special Issue 1), 85-95.

Barnes, A. S. (1939). The differences between natural and human flaking on prehistoric flint implements. American Anthropologist, 41(1), 99-112.

Binford, L. R. (1981). Behavioral archaeology and the "Pompeii premise." Journal of Anthropological Research, 37(3), 195-208.

Binford, L. R. (1992). Seeing the present and interpreting the past-and keeping things straight. In J. Rossignol y L. Wandsnider (Eds.), Space, time, and archaeological landscapes (pp. 43-59).

Binford, L. R. (2001). Where do research questions come from. American Antiquity, 66, 669-78.

Borrazzo, K., y Borrero, L. A. (2015). Taphonomic and archaeological perspectives from northern Tierra del Fuego, Argentina. Quaternary International, 373, 96-103.

Borrazzo, K. y Borrero, L. A. (2016). Caracterización del modo tafonómico en dunas de Fuego-Patagonia: aportes de la localidad Chorrillos (Tierra del Fuego, Argentina). Actas Del XIX Congreso Nacional de Arqueología Argentina, Facultad de Ciencias Naturales e IML, Universidad Nacional de Tucumán.

Borrazzo, K. (2006). Tecnología lítica del alero Cerro León 3 (Santa Cruz, Argentina). Magallania, 34(2), 63-74.

Borrazzo, K. (2010). Arqueología de los esteparios fueguinos. Tafonomía y tecnología lítica en el norte de Tierra del Fuego, Argentina. Tesis doctoral. Facultad de Filosofía y Letras, Universidad de Buenos Aires, Buenos Aires.

Borrazzo, K. (2011). Tafonomía lítica en la estepa patagónica: experimentación y registro arqueológico de superficie. En L. A. Borrero \& K. B. Borrazzo (Eds.), Bosques, montañas y cazadores: investigaciones arqueológicas en Patagonia Meridional (pp. 127-153). Buenos Aires: CONICET-IMHICIHU.

Borrero, L. A. (1991). Experimentos y escalas arqueológicas. Shincal, 3(1), 142.

Borrero, L. A. (2001). El poblamiento de la Patagonia: toldos, milodones y volcanes. Buenos Aires: Emecé.

Borrero, L. A. (2014a). Multi-service taphonomy: Shells, garbage, and floating palimpsests. Intersecciones en Antropología, (Special Issue 1), 13-20.

Borrero, L.A. (2014b). Regional taphonomy. En: Smith, C. (Ed.), Encyclopedia of Global 
Archaeology. Springer Reference, New York, pp. 72327235 .

Carranza Elola, J. (2015). Tecnología y tafonomía lítica del sitio quebrada Quereo: metodología para abordar conjuntos líticos ambiguos del pleistoceno tardio en la costa de Los Vilos. Tesis de licenciatura. (Escuela de Arqueología, Departamento de Antropología, Facultad de Ciencias Sociales, Universidad de Chile).

Carranza, M. E. (2015). Análisis de conjuntos líticos de superficie desde una perspectiva tafonómica. Estudios en la costa norte del golfo San Matías, Río Negro, Argentina. Tesis de Licenciatura. Facultad de Filosofía y Letras, Universidad de Buenos Aires, Buenos Aires.

Davies, B., Holdaway, S. J., y Fanning, P. C. (2016). Modelling the palimpsest: An exploratory agent-based model of surface archaeological deposit formation in a fluvial arid Australian landscape. The Holocene, 26(3), 450-463.

Dincauze, D. F. (1987). Strategies for Paleoenvironmental Reconstruction in Archaeology. In M. B. Schiffer (Ed.), Advances in archaeological method and theory (Vol. 11, pp. 255-336).

Domínguez-Rodrigo, M. (2008). Conceptual premises in experimental design and their bearing on the use of analogy: an example from experiments on cut marks. World Archaeology, 40(1), 67-82.

Dunnell, R. C. (1992). The notion site. In J. Rossignol y L. Wandsnider (Eds.), Space, time and archaeological landscapes (pp. 21-41). Nueva York: Springer Science+Business Media.

Eren, M. I., Durant, A., Neudorf, C., Haslam, M., Shipton, C., Bora, J., Korisettar, R. y Petraglia, M. (2010). Experimental examination of animal trampling effects on artifact movement in dry and water saturated substrates: a test case from South India. Journal of Archaeological Science, 37(12), 3010-3021.

Fanning, P. C., Holdaway, S. J., Rhodes, E. J., y Bryant, T. G. (2009). The surface archaeological record in arid Australia: Geomorphic controls on preservation, exposure, and visibility. Geoarchaeology: An International Jornal, 24(2), 121-146.

Flegenheimer, N., y Weitzel, C. (2007). Caminar sobre piedras: los artefactos fracturados de Cerro El Sombrero. In Actas del XVI Congreso Nacional de Arqueología Argentina (Vol. 3, pp. 263-267). Jujuy.

Gifford-Gonzalez, D. P. (1981). Taphonomy and paleoecology: a critical review of archaeology's sister disciplines. In M. B. Schiffer (Ed.), Advances in
Archaeological Method and Theory (Vol. 4, pp. 365-438).

Hiscock, P. (1985). The need for a taphonomic perspective in stone artefact analysis. Queensland Archaeological Research, 2, 82-97.

Lin, S. C., Rezek, Z., y Dibble, H. L. (2018). Experimental design and experimental inference in stone artifact archaeology. Journal of Archaeological Method and Theory, 25(3), 663-688.

Lopinot, N. H., y Ray, J. H. (2007). Trampling experiments in the search for the earliest Americans. American Antiquity, 72(4), 771-782.

Lyman, R. L. (1994). Vertebrate taphonomy. Cambridge University Press.

Mason, R. J. (1965). Makapansgat limeworks fractured stone objects and natural fracture in Africa. The South African Archaeological Bulletin, 20(77), 3-16.

Cañete Mastrángelo, D. S., \& Muñoz, A. S. (2018). Tafonomía de conjuntos arqueológicos en superficie: el caso de P 37 (desembocadura del río Santa Cruz), Patagonia meridional. Arqueología, 24(2), 161-176.

Méndez Muñoz, V. (2015). Historias Depositacionales de conjuntos líticos en la Transición Pleistoceno-Holoceno en el Sitio Valiente, Provincia del Choapa. (Tesis de grado.). Universidad de Chile, Chile.

Nami, H. G. (2011). Fundamentos teóricos y epistemológicos sobre arqueología y tecnología lítica experimental. Arqueología Rosarina Hoy, 75-98.

Nami, H. G. (2018). Theoretical and Epistemological Thoughts on Archaeology and Experimental Lithic Technology. Journal of Research in Philosophy and History, 1(2), 139-165.

Ozán, I. L. (2017). Gravity and the formation of the archaeological record: Main concepts and methodological tools. Geoarchaeology, 32(6), 646-661.

Rick, J. W. (1976). Downslope movement and archaeological intrasite spatial analysis. American Antiquity, 41(2), 133-144.

Schiffer, M. B. (1987). Formation processes of the archaeological record. Albuquerque: University of New Mexico Press.

Somonte, C., y Baied, C. A. (2013). Edad mínima de exposición de superficies en canteras-taller: reflexiones en torno a las primeras dataciones mediante microlaminaciones del barniz de las rocas (VML) para el noroeste argentino. Chungará (Arica), 45(3), 427-445. 
Ugalde, P. C., Santoro, C. M., Gayo, E. M., Latorre, C., Maldonado, S., De Pol-Holz, R., y Jackson, D. (2015). How do surficial lithic assemblages weather in arid environments? A case study from the Atacama Desert, northern Chile. Geoarchaeology, 30(4), 352-368.

Weitzel, C., Borrazo, K. B., Ceraso, A., y Balirán, C. (2014).
Trampling Fragmentation Potential of lithic artifacts: an experimental approach. Intersecciones en Antropología, 15(1), 97-110.

Wilk, R., y Schiffer, M. B. (1979). The archaeology of vacant lots in Tucson, Arizona. American Antiquity, 44(3), 530-536. 